\title{
Detailed simulations of Fermi-LAT constraints on UHECR production scenarios
}

\author{
Marco Muzio* \\ Center for Cosmology and Particle Physics, \\ Department of Physics, New York University, NY, NY 10003 USA \\ E-mail: msm659@nyu.edu

\section{Glennys R. Farrar} \\ Center for Cosmology and Particle Physics, \\ Department of Physics, New York University, NY, NY 10003 USA \\ E-mail: gf25@nyu.edu
}

\section{Michael Unger}

Institut für Kernphysik, Karlsruher Institut für Technologie, 76021 Karlsruhe, Germany

E-mail: Michael.Ungerekit.edu

\begin{abstract}
We have performed high-resolution simulations of the flux and spectrum of gammas detectable by Fermi-LAT, coming from UHECR-induced cascades, using the state-of-the-art (Gilmore) model of extragalactic background light, ELMAG to simulate the EM cascade (modified to work correctly for the Gilmore EBL) and CRPropa3 to simulate the production of EM secondaries from UHECR propagation. We examine the validity and limitations of the Liu et al. (2016) constraints, which found that we live in a local UHECR over-density. We also examine whether a model such as that of Unger, Farrar, and Anchordoqui (2015) - which explains the full extragalactic CR spectrum and composition including the ankle, based on nuclear photodisintegration in the neighborhood surrounding the source - satisfies the Fermi-LAT constraints, or requires Earth to be in an atypical position in the Universe as claimed by Liu et al. for pure proton primaries. We conclude that a mixed-composition scenario, like that of Unger, Farrar, and Anchordoqui, satisfies Fermi-LAT constraints evading any need for Earth to be in an atypical position in the Universe.
\end{abstract}

35th International Cosmic Ray Conference - ICRC2017

10-20 July, 2017

Bexco, Busan, Korea

* Speaker. 


\section{Introduction}

Ultrahigh-energy cosmic rays (UHECRs) produce gamma-rays through interactions with the intergalactic photon fields. Most notably, interactions of cosmic-ray protons produce electrons and positrons which can subsequently create high energy photons via inverse Compton scattering. The propagation of UHECRs through the intergalactic photon fields will therefore contribute to the isotropic gamma-ray background (IGRB), observable at Earth.

The extragalactic gamma-ray background (EGB) consists of emissions from resolved and unresolved point sources, like blazars, star-forming galaxies, and radio galaxies, as well as diffuse emissions due to cosmic ray (CR) propagation. The Fermi-LAT experiment, while also measuring the total EGB, has measured the IGRB in [1], which consists of unresolved extragalactic gammaray point sources and truly diffuse contributions (TDGRB) to the EGB. Using the source count distribution from [2], Liu et al. estimated the TDGRB to be $2 \times 10^{-8} \mathrm{GeV} \mathrm{cm}^{-2} \mathrm{~s}^{-1} \mathrm{sr}^{-1}$ in the 50 $\mathrm{GeV}$ energy bin [3]. Within the systematics of the source count distribution they derived a range between 0 and $4 \times 10^{-8} \mathrm{GeV} \mathrm{cm}^{-2} \mathrm{~s}^{-1} \mathrm{sr}^{-1}$. This estimate, therefore, provides an upper-limit on the truly diffuse photon contribution to the $50 \mathrm{GeV}$ bin of the Fermi-LAT IGRB spectrum. Liu et al. reached their conclusion on the need for a local UHECR overdensity ("fog") by comparing the predicted photon fluxes in a pure-proton source model to this upper-limit on the TDGRB [3]. However, it is well-known that the Pierre Auger Observatory (Auger) has discovered that the UHECR spectrum is composed of intermediate mass nuclei at energies above the ankle ( $E \gtrsim$ few EeV, [4], [5]), indicating that a pure-proton source model is not realistic. Therefore, in order to reproduce the UHECR spectrum and the composition observed by Auger, we must consider mixed-composition source models.

One such mixed-composition scenario is that of Unger, Farrar, and Anchordoqui ([6], UFA), which takes into account photonuclear interactions that take place between UHECRs and ambient photon fields in the source environment. By doing so, it has been shown that UFA models can beautifully reproduce the full extragalactic CR spectrum and composition [6]. Knowing this, we sought to investigate the photon fluxes predicted in UFA scenarios and evaluate the necessity of a "local fog" of UHECR sources in such a mixed-composition scenario.

\section{Analysis}

Our analysis takes advantage of the observation that for both CRs and electromagnetic (EM) cascades the average observed total (primary and secondary) flux on Earth depends only on the primary particle's initial energy, distance from Earth, particle type extragalactic background light (EBL) model used in simulations. By tabulating the observed fluxes at Earth for primaries whose energies and distances sufficiently sample the parameter space of interest, we are able to investigate various UHECR source models without performing any further simulations.

The tabulated fluxes used in this work were based on simulations performed using CRP ropa 3 (to simulate CR propagation, [7]) and ELMAG (to simulate EM cascades, [8]). Our simulations were performed using the Gilmore EBL ([9]) in a flat FRW cosmology. For CRs, the parameter space we considered was from $10^{17} \mathrm{eV}$ to $10^{20} \mathrm{eV}, 0 \mathrm{Mpc}$ to $6520 \mathrm{Mpc}$ in comoving distance from Earth ( $z \lesssim 3$ ), and atomic numbers from $A=1$ to $A=56$. In order to obtain a statistically accurate 
sampling of the observed flux of CRs, we used an injection distribution in each energy-distance bin with a uniform injection spectrum in $\log _{10} E$ and a uniform distribution in light-travel distance. In order for bins to be small enough so that our results were not very sensitive to the injection distribution inside each bin, our energy-distance bins were $20 \mathrm{Mpc}$ wide in comoving distance and 0.1 decades wide in energy (i.e. $\log _{10}\left(E_{i+1} / E_{i}\right)=0.1$ ).

In order to obtain the observed EM flux we needed to simulate EM cascades throughout the parameter space of interest and connect these cascades to the CR propagation which would have initiated them. To accomplish the latter, our CRPropa 3 simulations provided us with the distribution of EM secondaries produced throughout energy-distance space during CR propagation (see Fig. 1). For EM cascades, we considered a parameter space from $10^{8} \mathrm{eV}$ (the bottom of the FermiLAT range) to $10^{20} \mathrm{eV}$ and $0 \mathrm{Mpc}$ to $6520 \mathrm{Mpc}$ in comoving distance from Earth. EM particles were injected into energy-distance bins of the same size as those in the CR case and with the same injection spectrum and spatial distribution. From the observed EM fluxes due to EM cascades, the EM fluxes due to CR propagation were calculated by

$$
f_{i j}^{l}(E)=\sum_{m, n, k} N_{i j m n}^{k} f_{m n}^{k l}(E)
$$

where $f_{i j}^{l}(E)$ is the observed flux of EM particles of type $l\left(\gamma, e^{+}, e^{-}\right)$due to CR propagation initiated in the $i$ th energy and $j$ th distance bin, $N_{i j m n}^{k}$ is the number of particles of type $k$ produced in the $m$ th energy and $n$th distance bin due to CR propagation initiated in the $i j$ th energy-distance bin, and $f_{m n}^{k l}(E)$ is the observed flux of EM particles of type $l$ due to EM cascades initiated by a particle of type $k$ in the $m n$th energy-distance bin. All fluxes are normalized by the number of particles injected to produce the observed flux. In (2.1) we have suppressed indices labeling the CR species.

To find the observed CR and EM fluxes predicted by a given UHECR source model we perform a weighted sum

$$
f^{l}(E)=\sum_{i, j, A} w_{i j}^{A} f_{i j}^{A l}(E)
$$

where $f^{l}(E)$ is the observed flux of particles of type $l$ (now including EM particles and CRs) predicted by the given UHECR source model, $f_{i j}^{A l}(E)$ is the observed flux of particles of type $l$ due to propagation of CRs of nuclear species $A$ originating from the $i j$ th energy-distance bin, and $w_{i j}^{A}$ is the weight given to the $i j$ th energy-distance bin for nuclear species $A$ in then given UHECR source model.

In order to calculate the weights $w_{i j}^{A}$ we treat UHECR source models with the source itself as a blackbox. That is to say we do not consider the mechanisms for UHECR acceleration, their interaction with the source environment, or other source details. Our simulations represent CRs just outside the source environment, where they begin their propagation through the intergalactic medium. With this in mind a blackbox source model is fully characterized by three quantities: 1) the spatial distribution of sources throughout the universe, $\left.d N_{s} / d D ; 2\right)$ the composition output by the source, encoded by the relative abundance of each species $A, \alpha_{A}$; and 3 ) the injection spectrum of each nucleus, $d n_{A} / d E$. The integral of the first quantity, $d N_{s} / d D$, captures the number of sources in a given region measured in comoving distance, while the integral of the product of the latter two 


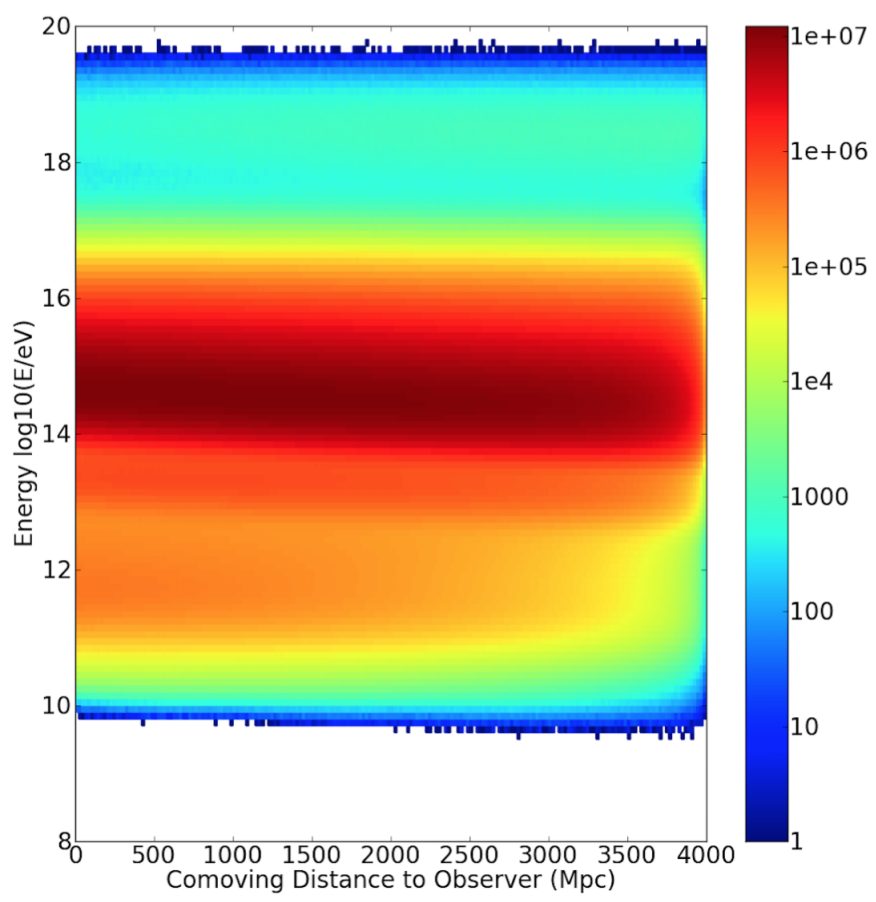

Figure 1: Distribution of the total number of EM secondaries produced during propagation to Earth for protons originating from the given energy-distance bin in the range of $0 \mathrm{Mpc}$ to $4 \mathrm{Gpc}$ in comoving distance and $10^{17} \mathrm{eV}$ to $10^{20} \mathrm{eV}$ in energy. Protons were injected into each energydistance bin with spectrum $d N / d E \propto E^{-1}$ and uniformly in light-travel distance. This table is a visual representation of $\sum_{i j} N_{i j m n}^{k}$ (cf. 2.1) for proton primaries, where the sum over $j$ runs from 0 Mpc to $4 \mathrm{Gpc}$ while the sum over $i$ runs over the entire energy range $10^{17}-10^{20} \mathrm{eV}$.

quantities, $\alpha_{A} d n_{A} / d E$, captures the number of CRs of nuclear species $A$ injected in a given energy range. Thus, the total number, $N_{i j}^{A}$, of CRs of nuclear species $A$ injected into a region from $D_{j}$ to $D_{j+1}$ in comoving distance with energies from $E_{i}$ to $E_{i+1}$ is given by

$$
N_{i j}^{A}=N_{0} \alpha_{A} \int_{E_{i}}^{E_{i+1}} \frac{d n_{A}}{d E} d E \int_{D_{j}}^{D_{j+1}} \frac{d N_{s}}{d D} d D
$$

and the total number of CRs injected in the $i j$ th energy-distance bin is given by

$$
N_{i j}=N_{0} \sum_{A} \alpha_{A} \int_{E_{i}}^{E_{i+1}} \frac{d n_{A}}{d E} d E \int_{D_{j}}^{D_{j+1}} \frac{d N_{s}}{d D} d D
$$

where we have defined $N_{0}$ as the total number of CRs injected throughout the universe (i.e. $N_{0}=$ $\sum_{i, j} N_{i j}$ ) and set $\sum_{A} \alpha_{A}=1, \int_{E_{\min }}^{E_{\max }} \frac{d n_{A}}{d E} d E=1$ for all $A$, and $\int_{D_{\min }}^{D_{\max }} \frac{d N_{s}}{d D} d D=1$. Our tabulated fluxes were calculated using a source model with a single nuclear species and $E_{\min }, E_{\max }, D_{\min }$, and $D_{\max }$ equal to the energy-distance bin edges.

In order for our source model to be made equivalent to a given UHECR source model, each energy-distance bin must be given a weight $w_{i j}^{A}$ so that the two models agree on the number of CRs of a given nuclear species $A$ injected bin-wise. Thus, the necessary weight $w_{i j}^{A}$ can be derived from (2.3): 


$$
\begin{aligned}
N_{i j}^{A} & =N_{0} \alpha_{A} \int_{E_{i}}^{E_{i+1}} \frac{d n_{A}}{d E} d E \int_{D_{j}}^{D_{j+1}} \frac{d N_{s}}{d D} d D \\
& =w_{i j}^{A} \overline{\alpha_{A}} \int_{E_{i}}^{E_{i+1}} \frac{\overline{d n_{A}}}{d E} d E \int_{D_{j}}^{D_{j+1}} \frac{\overline{d N_{s}}}{d D} d D
\end{aligned}
$$

where quantities on the right-hand side without the overbar correspond to those given by the desired UHECR source model and those with the overbar correspond to those used to calculate the tabulated fluxes. In light of the comment made above regarding the source model used to calculate the tabulated fluxes, (2.5) becomes

$$
w_{i j}^{A}=\alpha_{A} \int_{E_{i}}^{E_{i+1}} \frac{d n_{A}}{d E} d E \int_{D_{j}}^{D_{j+1}} \frac{d N_{s}}{d D} d D
$$

where we have set $N_{0}=1$. Thus, using our tabulated fluxes, (2.2), and (2.6) we are able to efficiently calculate the observed CR flux and observed EM flux predicted by a given blackbox UHECR source model.

\section{Results and Conclusions}

We began by verifying the findings of Liu et al. in a pure-proton source scenario. Liu et al. had concluded that a pure-proton scenario with a uniform source evolution was in tension with the upper-limit on the TDGRB, while a pure-proton scenario with a star formation rate (SFR) evolution was in conflict with this limit [3]. In our analysis we used an energy injection spectrum which produced a much better fit to the Auger spectrum of CRs [10] (see Fig. 2(a)). Specifically, we used an injection spectrum $d N / d E \propto E^{-2.66}$ in the case of a uniform source evolution and $d N / d E \propto E^{-2.47}$ in the case of a SFR source evolution. Here we fit to the 2013 data from Auger for consistency with UFA [6]. Our results are in good agreement with those of Liu et al.: we find that pure-proton scenarios are in tension or in conflict with the upper-limit on the TDGRB (see Fig. 2(b)).

We also investigated a pure-proton source scenario in which there is a low energy cutoff in the injection spectrum. For this we used the same injection spectrum as above and a hard cutoff at $E=$ $10^{18} \mathrm{eV}$. This reduces the predicted Fermi-LAT photon spectrum slightly, easing the tension for an SFR source evolution. Thus, the presence or abscence of a low energy component of protons makes no difference in the predicted Fermi-LAT spectrum. However, neither scenario can reproduce the composition of CRs observed by Auger.

Finally, we investigated a mixed-composition scenario, namely, the UFA fiducial scenario [6]. In this scenario we also took into account the photon flux coming out of the source due to photopion production in the source environment. The UFA fiducial model gives an excellent fit to both the observed flux of CRs (see Fig. 3(a)), as well as, the composition of CRs observed by Auger (cf. [6]). Morever, we note that the UFA fiducial scenario employs a SFR source evolution. We find that, despite using a SFR evolution, the fiducial UFA scenario is not in conflict with the TDGRB upper-limit (see Fig. 3(b)). 


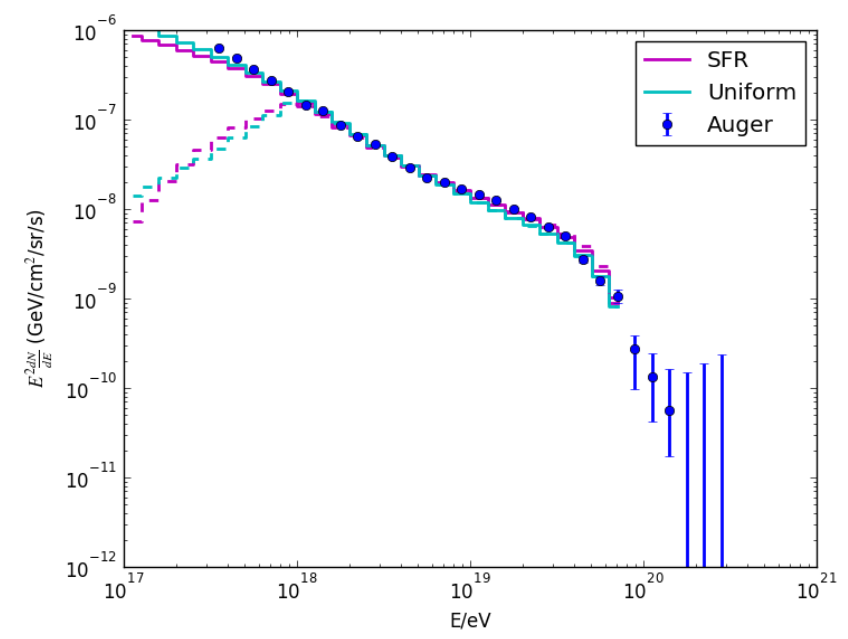

(a)

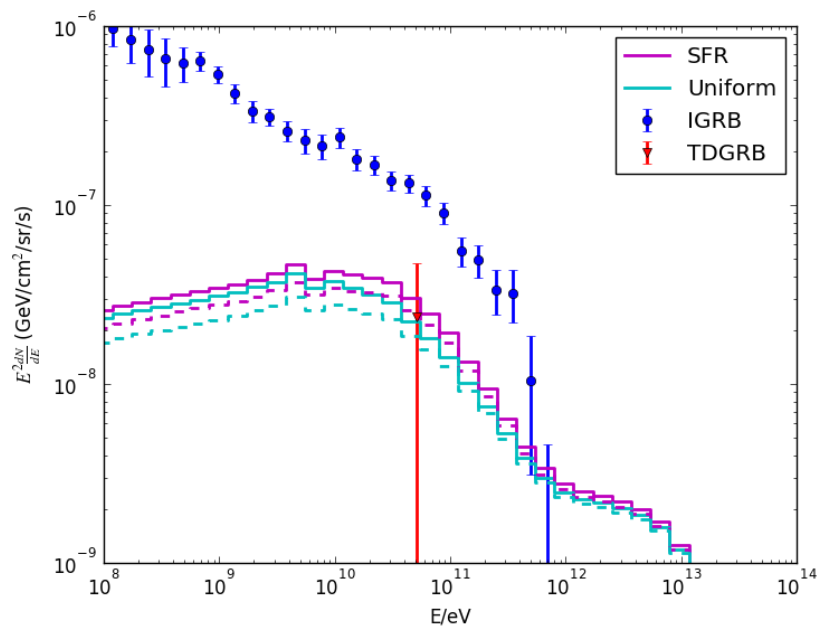

(b)

Figure 2: Predicted fluxes of (a) CRs and (b) photons in a pure-proton source scenario with SFR (magenta) and uniform (cyan) source evolutions. An injection spectrum with index $-2.47(-2.66)$ was used in the SFR (uniform) evolution scenario across the entire energy range (solid lines) and with a hard low energy cutoff at $10^{18} \mathrm{eV}$ (dashed lines). In (a) the CR spectrum observed by Auger is plotted in blue. In (b) the IGRB observed by Fermi-LAT is plotted in blue and the truly diffuse gamma-ray background (TDGRB) estimated in [3] from Fermi-LAT data [1], [2] is indicated with a red triangle. The red line gives the uncertainty range of this estimate. 


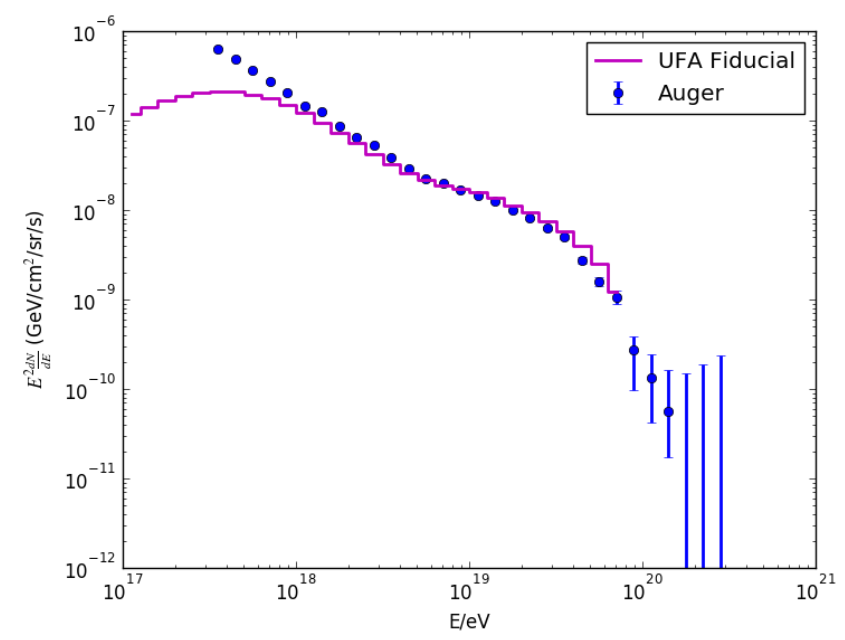

(a)

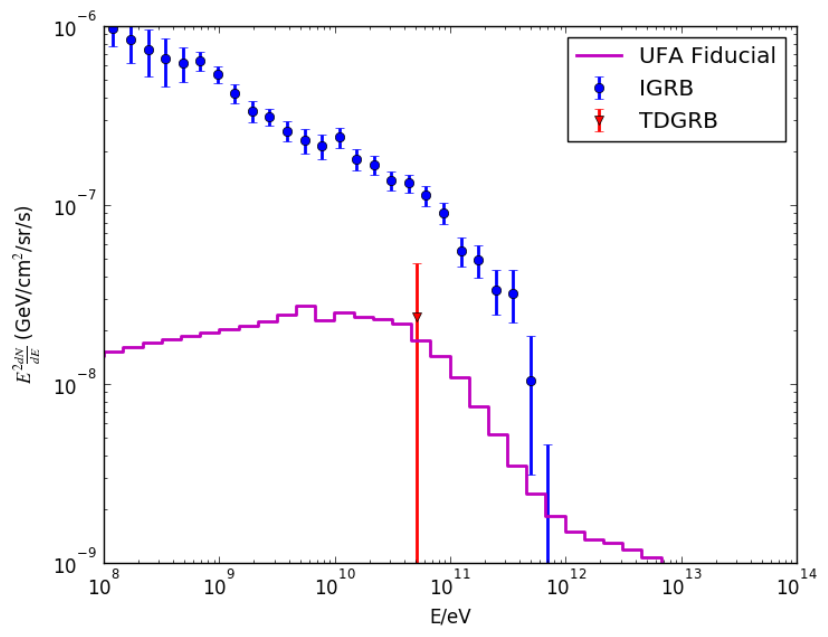

(b)

Figure 3: Predicted fluxes (magenta) in the UFA fiducial scenario (which has a mixed-composition and SFR evolution) of (a) CRs and (b) photons. In (a) the CR spectrum observed by Auger is plotted in blue. In (b) the IGRB observed by Fermi-LAT is plotted in blue and the truly diffuse gamma-ray background (TDGRB) estimated in [3] from Fermi-LAT data [1], [2] is indicated with a red triangle. The red line gives the uncertainty range of this estimate. 
The photon flux in the $50 \mathrm{GeV}$ energy bin represents approximately $87 \%$ of the upper-limit on the TDGRB. However, it is important to note that the UFA fiducial model is just one UFA scenario. Other UFA scenarios are known to produce lower fluxes of neutrinos than the fiducial scenario ([6]) and similarly variants on the fiducial scenario may produce lower fluxes of photons. This would allow for a wider breadth between the photon flux predicted in a mixed-composition scenario and the TDGRB upper-limit. Such scenarios will be explored in future work.

In this Proceeding we have shown that a mixed-composition scenario does not require a local overdensity of UHECR sources as proposed by Liu et al. Specifically, the diffuse photon flux predicted by the UFA fiducial scenario is not in conflict with the upper-limit on the TDGRB. Moreover, other UFA scenarios may produce even lower photon fluxes in energy bins above $50 \mathrm{GeV}$, further reducing any tension with data. A more detailed study of these scenarios and the sensitivity of photon fluxes to source evolution and source environment assumptions will be presented in a future publication.

\section{Acknowledgements}

The authors would also like to thank the 35th International Cosmic Ray Conference, and its organizers, for the invitation to present this work. MM would like to thank the New York University Physics Department for support throughout this work. The research of GRF is supported in part by the U.S. National Science Foundation (NSF), Grant NSF-1517319. MU acknowledges the financial support from the EU-funded Marie Curie Outgoing Fellowship, Grant PIOF-GA-2013-624803.

\section{References}

[1] Fermi-LAT Collaboration, M. Ackermann et al., Astrophys. J. 799 (2015) 86, arXiv:1410.3696.

[2] Fermi-LAT Collaboration, Phys. Rev. Lett. 116, 151105 (2016).

[3] Ruo-Yu Liu, Andrew M. Taylor, Xiang-Yu Wang, Felix A. Aharonian, Phys. Rev. D 94, 043008 (2016), arXiv:1603.03223.

[4] A. Aab et al. (Pierre Auger), Phys. Rev. D 90, 122005 (2014), arXiv:1409.4809 [astro-ph.HE].

[5] A. Aab et al. (Pierre Auger), Phys. Rev. D 90, 122006 (2014), arXiv:1409.5083 [astro-ph.HE].

[6] M. Unger, G.R. Farrar \& L.A. Anchordoqui, Phys. Rev. D 92 (2015) 123001, arXiv:1505.02153.

[7] R. Alves Batista, A. Dundovic, M. Erdmann, K.-H. Kampert, D. Kuempel, G. Mueller et al., JCAP 1605 (2016) 038, arXiv:1603.07142.

[8] M. Kachelriess, S. Ostapchenko, R. Tomas, Comput. Phys. Commun. 183 (2012) 1036-1043, arXiv:1106.5508.

[9] Gilmore R.C., Somerville R.S., Primack J.R., Domínguez A. (2012), Monthly Notices of the Royal Astronomical Society, 422, 3189, arXiv:1104.0671.

[10] A. Schulz, for the Pierre Auger Collaboration, 33rd ICRC, Rio de Janeiro, Brazil, 2013, arXiv:1307.5059 [astro-ph.HE]. 